\section{EPIDEMIOLOGICAL AND CLINICAL PATTERNS OF TRICHINELLOSIS IN BULGARIA FROM 1995 TO 2002}

\author{
PETKOVA S.*, MIHOV L.*, VUTOVA K.**, TSENOV I.***, \\ LA ROSA G.***** \& POZIO E.*****
}

Sir,

Trichinellosis is one of the most common helminthic zoonoses in Bulgaria. The number of infections has been incessantly on the rise during the last 17 years. From 1988 to 1998, 76 outbreaks were documented involving 6,770 people. Severe clinical courses inducing two deaths, were documented among 1,037 infected people (Kurdova et al., 1999). From 1998 to 2002, 1,804 cases of trichinellosis have been recorded in 118 outbreaks (Kurdova, 2001; Kurdova et al., 2003). From 1990 to 1992, clinical features of 117 patients with trichinellosis were studied at the Clinic of Parasitic Diseases of the Medical University of Sofia. The source of infection was uncontrolled pork from domestic pigs and wild boars in $59 \%$ and $41 \%$ of the infections, respectively (Boeva et al., 1995). The aim of the present study was to investigate the epidemiological and clinical patterns of people suffering from trichinellosis, who attended the Clinic of Infectious, Parasitic and Tropical Medicine of Sofia, Bulgaria, in the period 19952002.

Place of residence, age, type of dwelling of each enrolled person in the study was recorded. Most, but not all people under study, underwent clinical and epidemiological investigations including the duration of the incubation period, the clinical signs and symptoms and laboratory features (eosinophilia, leucocytosis, muscle enzymes, and circulating antibodies). Serology was performed by three commercial kits (Bul Bio NCIP Ltd, Bulgaria): passive haemagglutination assay (PHA), immunofluorescence (IF) and ELISA, according to the producer's instructions. Serum samples were collected from the enrolled persons from three to 46 days post

*Institute of Experimental Pathology and Parasitology, Bulgarian Academy of Sciences, Acad. Bonchev St., Block 25, Sofia, Bulgaria;

** Clinic of Infectious Parasitic and Tropical Medicine, Medical University, Sofia; *** Institute of Experimental Morphology and Anthropology, Bulgarian Academy of Sciences, Bulgarian Academy of Sciences, Sofia, Bulgaria; **** Department of Infectious, Parasitic and Immunomediated Diseases, Instituto Superiore di Sanita, Rome, Italy.

Correspondence: Dr Edoardo Pozio, Department of infectious, parasitic and immunomediated diseases, Istituto Superiore di Sanità, Viale Regina Elena 299, 00161 Rome, Italy.

Tel.: + 390649902304 - Fax. + 390649903561

E-mail: edoardo.pozio@iss.it infection. Trichinella sp. larvae were isolated from infected pork or pork products which were traced back as the source of the outbreak in the course of the epidemiological investigations, by $\mathrm{HCl}$-pepsin digestion according to a published protocol (Gamble et al., 2000) and identified by a multiplex-PCR analysis (Pozio \& La Rosa, 2003).

From 1995 to 2002, a total of 228 people with a suspected diagnosis of trichinellosis were enrolled in this study. Of them, 225 were diagnosed and treated for trichinellosis in the course of 18 outbreaks, whereas three were single cases. No death was documented. Both the outbreaks and the single cases were caused by the consumption of infected pork (raw minced meat or raw sausages) from backyard pigs or wild boars in $12(57 \%)$ and nine (43\%) foci, respectively (Table I). Of the 21 investigated foci, 12 (57\%) occurred in towns and nine (43\%) in villages (Fig. 1). The etiological agent was identified as T. spiralis in seven outbreaks and two single cases and as T. britovi in 11 outbreaks and in one single case. Trichinella britovi was the etiological agent of all infections caused by the consumption of pork from wild boars, whereas both T. spiralis (\# 9, $75 \%$ ) and T. britovi (\# 3, $25 \%$ ) were identified in pork from backyard pigs (Table I). No relationship was observed between the altitude of the place of origin of the infected animals and the etiological agent.

\begin{tabular}{lllrl}
\hline Year & $\begin{array}{c}\text { Source } \\
\text { of infection }\end{array}$ & $\begin{array}{c}\text { Trichinella } \\
\text { species }\end{array}$ & $\begin{array}{c}\text { No. of } \\
\text { infected } \\
\text { persons }\end{array}$ & Locality \\
\hline 1995 & Wild boar & T. britovi & 50 & Godech \\
1995 & Backyard pig & T. spiralis & 20 & Strelcha \\
1995 & Wild boar & T. britovi & 17 & Rakovitsa \\
1995 & Wild boar & T. britovi & 3 & Ichtiman \\
1996 & Wild boar & T. britovi & 27 & Samokov \\
1996 & Backyard pig & T. britovi & 7 & Kovachevtsi \\
1996 & Backyard pig & T. spiralis & 1 & Botevgrad \\
1996 & Backyard pig & T. britovi & 7 & Strelcha \\
1997 & Backyard pig & T. spiralis & 10 & Pernik \\
1997 & Backyard pig & T. britovi & 14 & Nova Zagora \\
1997 & Backyard pig & T. spiralis & 7 & Razgrad \\
1998 & Backyard pig & T. spiralis & 20 & Kiryaevo \\
1998 & Wild boar & T. britovi & 3 & Kustendil \\
1999 & Wild boar & T. britovi & 13 & Etropole \\
1999 & Backyard pig & T. spiralis & 3 & Mokresh \\
2000 & Wild boar & T. britovi & 15 & Borovets \\
2000 & Backyard pig & T. spiralis & 2 & Novi Iskar \\
2001 & Backyard pig & T. spiralis & 4 & Sofia \\
2001 & Wild boar & T. britovi & 3 & Dragalevtsi \\
2001 & Wild boar & T. britovi & 1 & Negovan \\
2002 & Backyard pig & T. spiralis & 1 & Yardjalovtci \\
\hline
\end{tabular}

Table I. - Epidemiological data of 18 outbreaks and three single cases of trichinellosis detected in Bulgaria from 1995 to 2002. 


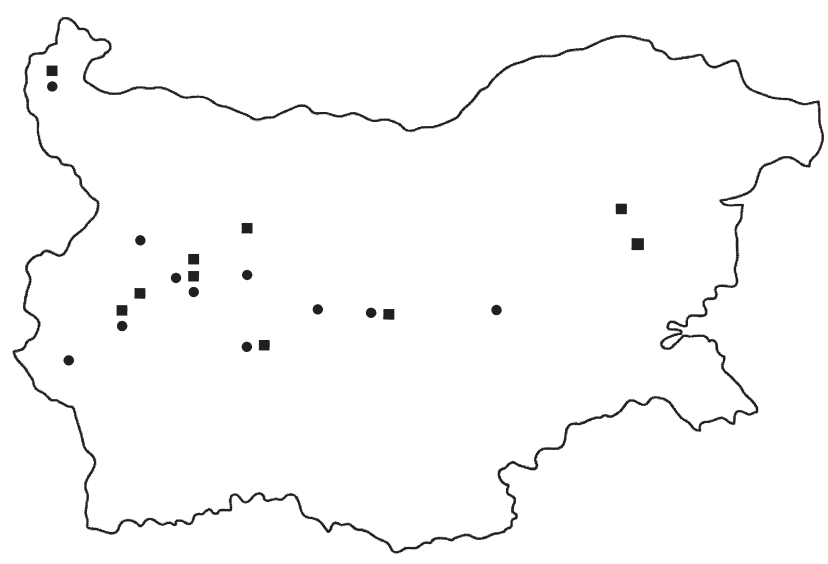

Fig. 1. - Map of Bulgaria with the place of origin of animals infected with Trichinella spiralis $(\square)$ or Trichinella britovi $(\mathbf{)}$ which were the source of infection for humans between 1995 to 2002 .

Of 228 enrolled people (112 males, 116 females; age range 1-70 years and three over 70 years), 160 (70 \%) were infected by T. britovi and 68 (30\%) by T. spiralis. Most of the enrolled people were positive by one or more serological tests. However, only 169 patients displayed the clinical picture of trichinellosis by one, two or more signs and/or symptoms. The other 59 people were asymptomatic, although all of them displayed a positive serology. Periorbital oedema was observed more frequently in T. spiralis than in T. britovi infected patients ( $\mathrm{p}<0.001)$. By contrast, weakness, diarrhoea and facial oedema were more frequently observed in T. britovi infected patients (Table II). No difference was

\begin{tabular}{lcc}
\hline Clinical features & $\begin{array}{c}\text { T. britovi (105) } \\
\text { No. (\%) }\end{array}$ & $\begin{array}{c}\text { T. spiralis (64) } \\
\text { No. (\%) }\end{array}$ \\
\hline Muscle pain & $105(100)$ & $51(80)$ \\
Fever $\left(\geq 38^{\circ}\right.$ C) & $42(40)$ & $54(84)$ \\
Periorbital oedema & $56(53)$ & $58(90)$ \\
Facial oedema & $105(100)$ & $25(39)$ \\
Weakness & $26(25)$ & $20(31)$ \\
Diarrhoea & $26(25)$ & $20(31)$ \\
Rash & $38(36)$ & $19(30)$ \\
Conjunctivitis & $16(15)$ & $11(17)$ \\
\hline
\end{tabular}

\begin{tabular}{lcl}
\hline Laboratory features & & \\
Eosinophilia & $105(100)$ & $59(92)$ \\
eucocytosis & $84(82)$ & $45(70)$ \\
$\mathrm{CK}^{1}>80 \mathrm{U} / 1$ & $96(91)$ & $43(67)$ \\
$\mathrm{LDH}^{2}>240 \mathrm{U} / 1$ & $105(100)$ & $48(75)$ \\
\hline
\end{tabular}

${ }^{1}$ normal values 2-80 U/1; ${ }^{2}$ normal values 120-240 U/1.

Table II. - Number and percentage of symptomatic people showing clinical and laboratory features, who attended the Clinic of Infectious, Parasitic and Tropical Medicine, Sofia, Bulgaria, in the period 1995-2002. observed between T. spiralis and T. britovi infected patients for muscle pain, rash, conjunctivitis, leukocyte and eosinophil counts, and muscle enzyme (CK and $\mathrm{LDH})$ levels. The body temperature values ranged between $38-40^{\circ} \mathrm{C}$ in $83 \%$ and $42 \%$ of T. spiralis and T. britovi infected patients ( $\mathrm{p}<0.001)$; however, $47 \%$ of T. spiralis infected persons had values $\geq 39^{\circ} \mathrm{C}$ versus $11 \%$ of $T$. britovi infected persons. Of 228 enrolled persons with suspected trichinellosis, only 105 infected with T. britovi and 64 with T. spiralis showed clinical symptoms suggestive of trichinellosis (Table II). In T. spiralis infected patients, an incubation period of $16-$ 20 days was observed in $50 \%$ of people, while $24 \%$ of the T. britovi infected people showed an incubation period of 10-15 days. In general, T. spiralis infected persons showed a shorter incubation period than those infected by T. britovi. All 228 patients were treated with mebendazole (20 mg/kg for 10-14 days); of them, seven with a severe clinical picture resembling an intoxication syndrome with ecchymoses, were also treated with corticosteroids (prednisolone, $4 \mathrm{mg}$ three times a day for 5-10 days). Other patients with a milder symptomatology were treated with clemastin fumarate (1 mg, two times a day for 5-10 days) or diclofenac natrium (25 mg, three times a day for 5-10 days).

The occurrence of 18 outbreaks and single cases in Bulgaria between 1995 and 2002 shows that trichinellosis is still endemic in this country and pork from backyard pigs and wild boars continues to be the source of infection because the veterinary services do not test game animals and backyard pigs. The identification of the etiological agent as T. spiralis and T. britovi confirms previous reports on the presence of these two species in Bulgaria (Kurdova, 2001). The detection of only $T$. britovi in wild boars shows that this species is prevalent in wildlife but, when humans fail to properly breed domestic pigs this parasite reaches the domestic environment as registered in three outbreaks caused by the consumption of pork from backyard pigs. The present data suggest that in Bulgaria T. spiralis is restricted to the domestic habitat; however, there is a report showing that this parasite species is also present in wildlife of this country (Kurdova et al., 2004). In agreement with most of literature data (Dupouy-Camet et al., 2002; Pozio et al., 2003), in the course of these outbreaks and single cases, no information was available on the infective dose. It follows that the evaluation of the pathogenicity of the Trichinella species is very difficult; however, some data suggest, at least in part, that T. spiralis is more pathogenic than T. britovi. In fact, patients infected with $T$. spiralis displayed a higher body 
temperature for a longer period of time than those infected with $T$. britovi.

According to Pozio et al. (1993), T. spiralis and T. britovi show, at least in part, two distinct clinical patterns in the course of the disease: people infected with T. spiralis displayed specific IgG for a longer time, higher levels of CPK and more severe intestinal symptoms than those infected with $T$. britovi. The higher virulence of T. spiralis in comparison with that of T. britovi seems to be related to the female fecundity which is twice more prolific in T. spiralis than in T. britovi (Britov \& Figurnov, 1984). However, the pathogenicity role of Trichinella species remains to be elucidated, and certainly warrants further investigations. In conclusion, the continuous occurrence of human trichinellosis in Bulgaria is related to the disappearance of the large industrialised pig farms and to the increase of the number of backyard pigs which, together with wild boars, escape veterinary controls.

The work has been supported by Bulgarian National Science Foundation (Agrarian Commission - project CC1512/2005) and by the MED-VET-NET project of the European Union (WP27, Trichimed).

\section{REFERENCES}

Britov V.A. \& Figurnov V.A. Trichinellosis in Human and Animals of the Far East in the USSR. Pacific subdivision of the Far East Zonal Research Veterinary Institute, Vladivostok, 1984, 48 p (in Russian).

Boeva V., Vuchev D., Jeleva R., Stoicheva E., Vutova K. \& Dimova I. On the clinical and the epidemiological features of some trichinellosis with different sources of invasion. Savremenna medicina 1995, 4, 10-13 (in Bulgarian).

Dupouy-Camet J., Kociecka W., Bruschi F., Bolas-Fernandez F. \& Pozio E. Opinion on the diagnosis and treatment of human trichinellosis. Expert Opinion in Pharmacotherapy, 2002, 3, 1117-1130.

Gamble H.R., Bessonov A.S., Cuperlovic K., Gajadhar A.A., van Knapen F., Noeckler K., Schenone H. \& Zhu X. International Commission on Trichinellosis: recommendations on methods for the control of Trichinella in domestic and wild animals intended for human consumption. Veterinary Parasitology, 2000, 93, 393-408.

Kurdova R., Petrov P. \& Jordanova D. Human trichinosis in Bulgaria (1988-1998). Microbiologia Balcanica 99, First Balkan conference of microbiology, Plovdiv, Bulgaria, October 5-9, 1999, p. 201.

KuRdova R. New trends in parasitism in Bulgaria. Trends in Parasitology, 2001, 17, 314-315.

Kurdova R., Jordanova D., Vutchev D., Filippov G., Rainova I, Charizanov R., Dimitrov C., Ivanova M. \& Marinova T.
Status, surveillance and control of indigenous and imported parasitoses in Bulgaria. NCIPD Information Journal, 2003, 5, 5-29 (in Bulgarian).

Kurdova R., Muller N., Tsvetkova N., Mihov L., Georgieva D., IVANOVA M. \& GotTSTEIN B. Characterization of Trichinella isolates from Bulgaria by molecular typing and crossbreeding. Veterinary Parasitology, 2004, 123, 179-188.

PozIo E. \& LA Rosa G. PCR-derived methods for the identification of Trichinella parasites from animal and human samples. Methods in Molecular Biology, 2003, 216, 299-309.

Pozio E., Gomez Morales M.A. \& Dupouy-Camet J. Clinical aspects, diagnosis and treatment of trichinellosis. Expert Review Anti Infective Therapy, 2003, 1, 471-482.

Pozio E., Varese P., Gomez Morales M.A., Croppo G.P., PelLICCIA D. \& BRusChI F. Comparison of human trichinelloisis caused by Trichinella spiralis and by Trichinella britovi. American Journal of Tropical Medicine and Hygiene, 1993, $48,568-575$.

Reçu le 17 septembre 2007 Accepté le 26 décembre 2007 